\title{
Identification and Classification of numerous leaves diseases with Pattern Recognition Technique
}

\author{
Geetu Yadav \\ Student, Electronics and communication Department,, \\ Punjabi University,Patiala, India \\ Dipti Bansal \\ Asst. professor, Electronics and communication Department, \\ Punjabi University, Patiala, India
}

\begin{abstract}
Plant diseases are major reason for huge harm and economic losses in agricultural field. Identification of disease in the plant is very serious for balanced farming. Farmers faces major difficulties in testing the disease and also worried about the cost involved in this to compensate loss. The main aim of proposed work is to correctly identify leaves diseases with the use of neural networks in which image is converted into Lab color space .Segmentation is done by $k$ means clustering to spot diseased part of leaf after this GLCM texture feature are extracted. On the basis of feature selection classification is performed by neural pattern recognition tool with $96.9 \%$ correctness.
\end{abstract}

Keywords - K-means clustering,Neural pattern recognition tool and GLCM feature extraction

\section{INTRODUCTION}

The greater part of our nation are relied on the farming and yield production. Farming is source of employment for large number of people in our country because $70 \%$ of people work in this field that implies it nourishes the abundant number of individuals. But in this various diseases affected this farming due to many reasons. Mainly three types of diseases occur in plant leaves that is bacterial, fungus and viral. These are harmful for plants because it reduces its production and quality leads to economic loss.[1]so to detect these diseases earlier methods are naked eye opinion but this method is not so accurate needs experts advice.

Various image processing techniques have been applied to identify the various leaves diseases. For examples artificial neural network are used in characterization of orchid seed disease identification on the basis of Co occurrence matrix and its feature selection after conversion in grey scale.[2]Back propagation is applied ]to lemon disease in leaf. Feature are extracted from co occurrence matrix and HSI color space then discriminant static algorithm have been applied for characterization of disease.[3]

In this manner searching for quick, less costly and exact strategy to recognize and classify the diseases from the symptoms that occurred in leaves. Numerous framework has been proposed to reduce the problem occurring in farming business by making use of image processing and classification tools.

\section{RELATED WORK}

Santanu Padhikar and Jaya Sil [4]analyze the rice disease by various pattern recognition techniques.For segmentation, image brightness is enhanced by its conversion into HIS (Hue,Intensity and Saturation)model and then depending on entropy bi-level thresholding has been applied.With the help of thresholding disease spots are identified.SOM(Self Organizing Map) for classification in which grey spot of diseased part is fed as input.spots are made of uniform size with zooming algorithm then unsupervised SOM is used.After 50 epochs of training for 300 input dataset $92 \%$ accuracy has obtained.S.Anami et.al.[5] has done classification based on ANN(Artificial Neural Network) and BPNN(Back Propagation Neural Network) for various vegetables and cereals. For this, they converted 
image into HIS and features are extracted.Classification firstly done on the basis of color feature with which $80 \%$ precision has been obtained for normal vegetable and $85 \%$ for affected.Based on texture feature overall accuracy $78 \%$ has been obtained and $84 \%$ by combining them both.P.R.Rothe and R.V.Kshirsagar [6] presents pattern recognition system for cotton leaf disease like alterneria,bacterial blight and Myrothecium.Gaussian filter is used for preprocessing which averages the current pixels with respect to neighboring pixels. Snake segmentation is for finding out diseased portion and then seven hue components for feature extraction and based on features classification is performed using backpropagation with $85.52 \%$ exactness.Muthukannan et.al.[7] in this they have used bitter gourd leaf and bean leaf for identification and classification using FFNN(Feed Forward Neural Network),LVQ(Linear Vector Quantization) and RBF(Radial Basis Function) on the basis of texture features $90.7 \%$ precision for FFNN ,56.77\% for LVQ and $71.18 \%$ for RBF achieved.A.Gulhane et.al[8] identified diseases in cotton leaves with the help of MSOFM and SOFM.Leaf segmentation is done by unsupervised MSOFM(Modified Self organizing feature maping) and further lesion by Modified SOFM.color segments are obtained and trained with support vector machine For number of color groups genetic algorithm is used.Mengistu .et.al[9] designed a model for coffee leaves diseases identification in which initially preprocessing of image is done with some filtering tools then segmentation with kmeans clustering.Principal component analysis(PCA) and genetic algorithm applied to reduce big matrices of GLCM and color feature into small size.Next classification by KNN,ANN,SOM and RBF with correctness of $58.16 \%, 79.04 \%, 53.47 \%$ and $90.07 \%$. Girish Athainikar and Priti Badar[10] calculated mean, variance and range of both RGB and HSV color features and GLCM texture feature for classification by Back Propagation Neural Network(BPNN).with the help of image processing bioinformatics toolbox $92 \%$ accuracy obtained for potato leave diseases like early blight,roll viral and insect damage.

\section{PROPOSED METHODOLOGY}

We proposed a system which is helpful to identify leaves diseases and lead to increase in production and quality in farming system. We have used Pattern recognition tool for classification of diseases in which we test the data with trained data and indicates type of disease with which it is affected.

\section{A.IMAGE PREPROCESSING}

Image preprocessing in which test image background noise is removed and contrast is enhanced it means simply to highlight the features of interest. It is subjective procedure to manipulate image to take gain of visual aspects of human visual system.

\section{B.LAB SPACE TRANSFORMATION.}

It is used matching the luminance of greyscale to color scale.After getting the RGB values it encodes this intensity with gamma expansion.RGB image is converted into Lab color space in which L stands for luminosity layer and a for chromaticity layer says about color failure along red-green axis and b chromaticity layer color failure along blueyellow axis.

\section{C.K-MEANS CLUSTERING ALGORITHM}

It is unsupervised learning algorithm and important step to find out the diseased portion in leaf image. In this algorithm image is divided into certain number of cluster to find out centroid of each cluster. These centroid help us indicating different location of same image.

\section{D.GLCM FEATURE EXTRACTION}

Grey Level Co occurrence Matrix(GLCM) in this texture of diseased cluster is taking into consideration. Before this image is converted into greyscale for feature extraction. It is the statistical way to describe shape by sampling certain 
grey levels that occur with relationship other grey levels. It measures probability that one particular grey level will occur at a different distance and orientation from any pixel that has second grey level. Here we compute Energy,Entropy,Contrast,CorelationHomogenity,Smoothness,Kurtosis and IDM.From these feature selection leaves disease are classified.

\section{E.NEURAL NETWORK PATTERN RECOGNITION TOOL}

At last ,classification is performed for leaves diseases with Pattern recognition toolbox. Pattern recognition is implemented by multilayer feed forward neural network and trained with back propagation algorithm, in this when input is given output response is compared with desired response then error is calculated on which weights are adjusted.

\section{RESULT}

In Neural network approach input data is given in the form of matrix of 32 samples of in which 8 of each four classes Alterneria,Ascochyta,Target spot and Downy mildew are given to toolbox. Target data is in the form of binary that is 0 and 1.Fig. 1 shows the Network for data.

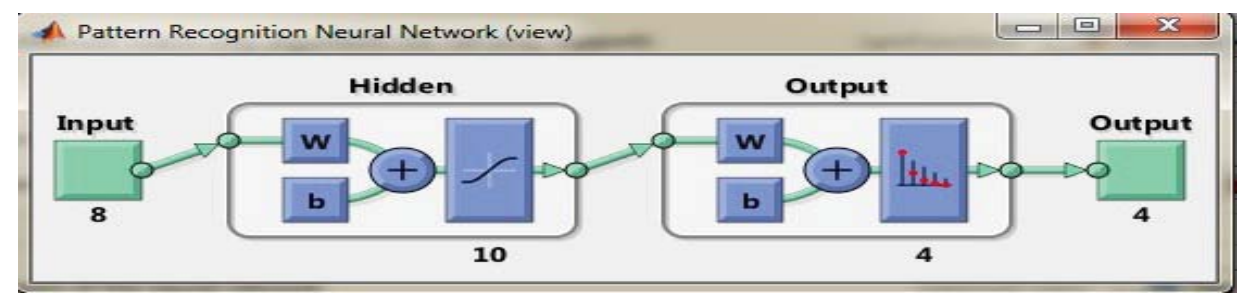

Fig.1Pattern Recognition Network

After putting input and target data,training is done with conjugate gradient backpropagation in pattern recognition toolbox in MATLAB 2015a in which 70\% data used for training and 15\% for testing and validation each. In Fig. 1 Neural network box after 35 epochs based on training of scaled conjugate gradient confusion matrix ,error histogram and performance are obtained.Fig. 2 shows accuracy of classifier by confusion matrix for various classes. 


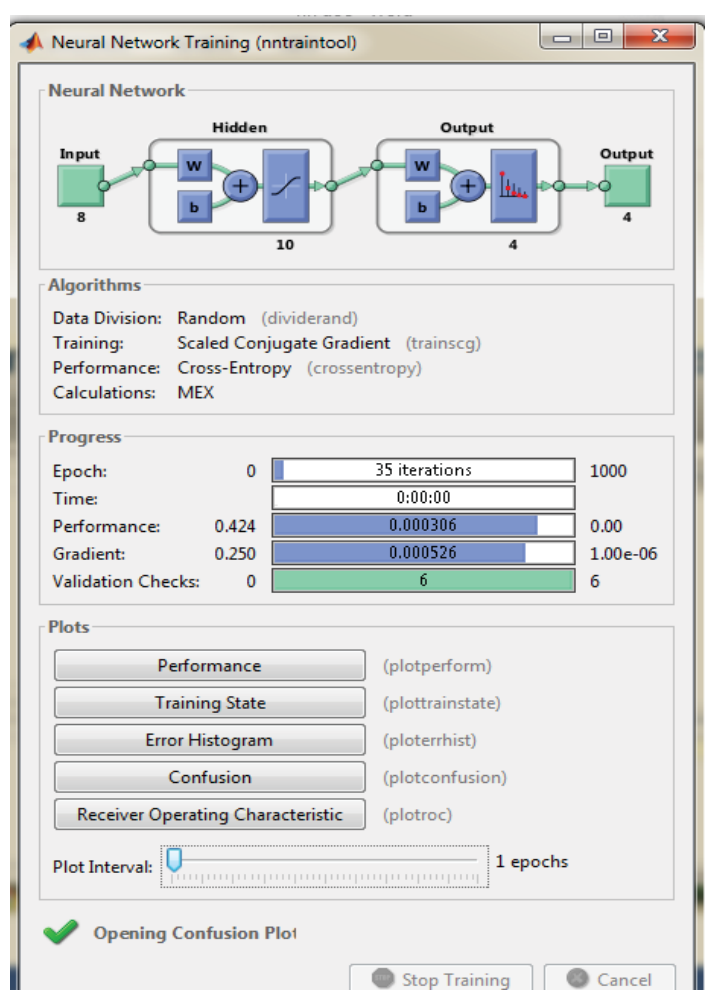

Fig.2 Neural Network box

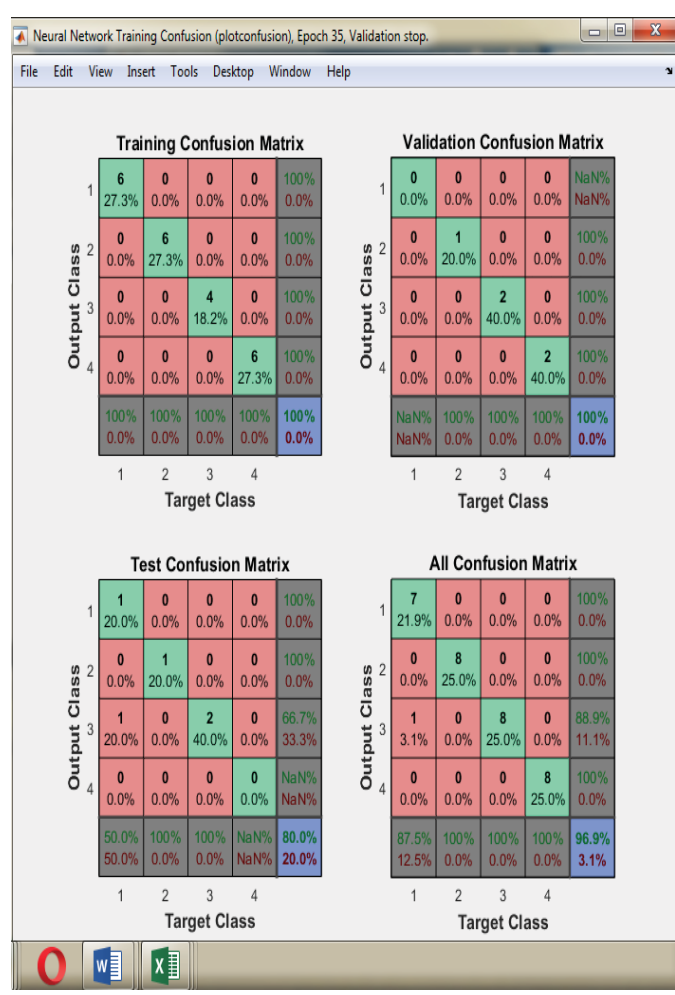

Fig.3 Confusion matrix for all,training,test and validation classes

For 32 samples of leaves accuracy for all $96.9 \%$ has been obtained ,100\% for training and validation and $80 \%$ for testing.In training input set is analyzed with known output responses ,if there is some error then trained again or error sent back to adjust weights and to get good results. Identification of disease is done by comparing the features value of training with testing.after testing result obtained is shown in Fig.4 that is infected disease is alterneria.

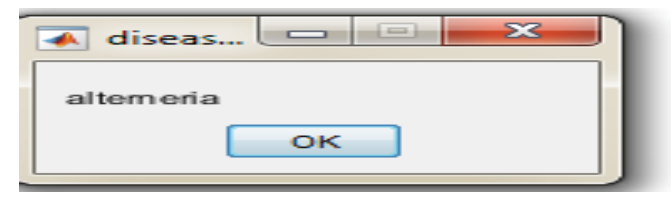

Fig.4 Result box

\section{CONCLUSION}

The precise recognition is vital. This paper speaks to testing the leaves diseases for better accuracy with classification with neural networks. We have used GLCM features extraction for diseased portion after its segmentation by K-means clustering method. In future to enhance the rate we can use hybrid method of various machine learning algorithms like SVM(Support Vector Machine), fuzzy logic and other clustering methods.

\section{REFERENCES}

[1] Rajleen kaur and Sandeep singh kang, “An enhancement in classifier support vector machine to improve plant disease detection"IEEE third international conference on MOOCs,2015 pp135-140.

[2] HuangK .Y., "Application of artificial neural network for detecting phalaenopsis seedling diseases using color and texture feature" Computers and Electronics in Agriculture,2007,pp3-11. 
[3] Pydipati,R.,Burks T.F. and Lee,W.S. ,"Identification of Citrus disease using color texture features and discriminant analysis" Computers and Electronics in Agriculture,2006,pp49-59.

[4] Santanu Padhikar and Jaya Sil, "Rice disease identification using pattern recognition techniques"IEEE eleventh international conference on ICCIT, December 2008 , pp420-423.

[5] Basvaraj .S.Anami,J.D.Pujari and Rajesh Yakkunadimathantanu ,"Identification and classification of normal and affected agriculture/horticulture produce based on combined color and texture feature extraction "International journal of computer applications in engineering science,2011,pp356-360.

[6] P.R.Rothe and R.V. Krishsagar "“Cotton leaf disease identification using pattern recognition using pattern recognition techniquesl”IEEE international conference on ICPC,2015,pp1-6.

[7] K.Muthukannan,P.Latha,R.Pon.Salvi and P.Nisha Honghai Yang et.al. Honghai Yang, S. Khandekar, M. Groll, "Classification of diseased plant leaves using neural network algorithms",ARPN journal of engineering and applied science,2015,pp1913-1919.

[8] Viraj .A.Gulhane and Ajay .A.Gurjar Meena, "Detection of diseases on cotton leaves and its possible diagnosis" International journal of image processing,2011.pp590-598.

[9] Abrham Debasu Mengistu,Degnachew Melesew Alemayhue and Seffi Gebeyehue Mengistu "Ethiopian coffee plant disease recognition based on imagining and machine learning techniques"International journal of database theory and applications,2016,pp79-88.

[10] Girish Athainikar and Priti Badar, "Potato leaf diseases detection and classification system" International Journal of mobile computing, 2016,pp76-88, ISSN 2320-088X. 